\title{
FURTHER EVIDENCE FOR GENETIC INFLUENCES ON EDUCATIONAL ACHIEVEMENT
}

\author{
BY C. E. GILL \\ (Biometrics Unit, University of Western Australia) \\ R. JARDINE \\ (Research School of Biological Sciences, Australian National University) \\ AND N. G. MARTIN \\ (Department of Human Genetics, Medical College of Virginia, USA)
}

\begin{abstract}
Summary. A genetic analysis of Tertiary Admission Examination (TAE) and Australian Scholastic Aptitude Test (ASAT) results for 264 pairs of MZ and DZ twins is reported. Purely environmental models are rejected as inadequate explanations of variation in examination performance and genetic factors must be invoked to obtain a satisfactory fit to the data. Within the portion of the age cohort who are candidates for these examinations, genetic factors appear to account for about 70 per cent of variation while environmental experiences shared by siblings appear to have little or no influence. However, when corrections are made on the assumption that examination candidates represent the top 34 per cent from a normal distribution of ability in the population, much greater variation between families is inferred for the population. If we also take account of the high correlation in educational achievement between husbands and wives the putative population twin correlations are consistent with heritabilities between 0.6 and 0.7 and modest contributions of shared environment around 20 per cent of the total variance. The data suggest that a distinction between IQ tests and tests of scholastic achievement on the basis of their causes of variation is not justified. We also show that while a common genetic factor is responsible for much of the covariation of ASAT Arts and Science scores, there are also some differences in the genes responsible for variation in the two areas.
\end{abstract}

\section{INTRODUCTION}

IN the last decade there have been great strides forward in the development of efficient methods for the analysis of individual differences and in their application to cognitive abilities. There is now little doubt that genetic factors contribute significantly to individual differences in intelligence and educational achievement (Eaves, 1975; Rao et al., 1977; Rice et al., 1980; Horn et al., 1982) although it has been suggested that the heritability for scholastic achievement is lower than that for IQ (Jensen, 1969). Of far greater moment than the precise value of the heritability, however, are issues frequently overlooked in the traditional literature on the genetics of cognition. Is there evidence for environmental influences shared by siblings which would support the supposed importance of home background and differences between schools? Are people with different genes for ability equally influenced by environmental factors such as teaching methods (genotypes $\times$ environments interaction)? Is all genetic variation additive so that children's abilities can, on average, be predicted from parents' abilities, or is there non-additive genetic variation which makes such a prediction less accurate? What is the effect on ability differences between families of the well-known tendency of people to marry spouses of ability similar to themselves?

The most powerful method for beginning to answer some of these questions is the classical twin study in which monozygotic (MZ) and dizygotic (DZ) twins are compared. Martin (1975), analysing the South Australian Intermediate Examination results of 149 pairs of twins aged 14-15 years, found heritabilities in the range $0.76-0.89$ for performance in mathematics, the sciences, languages and geography. He concluded 
that educational achievements, at least as assessed in large scale testing programmes, have heritabilities in the same range as IQ. One puzzling feature of Martin's results, however, was the absence of any evidence for the between pairs component of variation which one would have expected if cultural transmission and assortative mating were making any contribution to variation in educational achievement, as they do for IQ (Fulker and Eysenck, 1979).

In most studies of educational achievement the less able portion of the cohort escapes ascertainment (Martin, 1975; Loehlin and Nichols, 1976). Martin and Wilson (1982) have shown that one-sided sample selection of this kind can cause considerable bias in the estimation of genetic and environmental components of variance which are applicable to the population as opposed to the truncate sample. When they applied a correction to Martin's (1975) results to allow for the fact that only the top 67 per cent of the cohort were examined, estimates of population variance were now consistent with the existence of between families variance attributable to assortative mating or cultural transmission.

In the present study we analyse examination results in a sample of 17 -year-old twins in which only the most able 34 per cent of the age cohort are represented. We attempt to take account of this severe truncate selection of the twin sample and also of the well-known correlation between husbands and wives for educational attainment in our analysis of the causes of individual differences in examination performance. We show that once allowance is made for these two complications the breakdown of variance is very similar to that found for IQ.

A further issue is the cause of correlation between ability scores. Two views may be advanced which are related to the classic debate between Spearman and Guilford on the factor structure of IQ. At one extreme is the view that all correlations are caused by a common set of genes which determine general ability and that it is environmental influences which channel this ability towards excellence in one subject or another. At the other extreme are envisaged independent gene effects, each responsible for variation in a different ability while environmental circumstances cause correlation between them by moving all scores up or down in concert. In this paper we attempt to throw light on this issue by analysing the causes of covariation between scores on an Arts and a Science test.

\section{SAMPLE AND MEASUREMENTS}

Each year approximately 8700 final year high school students (average age 17 years), or 34 per cent of the age cohort, attempt the Tertiary Admissions Examination (TAE) in Western Australia. There is a choice of 35 subjects in which a candidate may be examined although many of these are attempted by very few people. Candidates attempting the TAE normally enter for six subjects, with a small number attempting seven or more. Since 1976, most candidates have also completed the Australian Scholastic Aptitude Test (ASAT), developed by the Australian Council for Educational Research, which yields scores for performance in Mathematics/Science (ASAT Science) and Humanities/Social Science (ASAT Arts). To ensure comparability between examination results in different subjects, TAE scores are scaled by an anchor variable method, whereby the raw scores of a group taking a particular subject are scaled according to their performance on the ASAT test.

Through the Tertiary Institutions Services Centre, which administers the TAE, the magnetic tapes containing the TAE and ASAT results of all candidates in the period from 1975 to 1981 were used to extract candidates with the same surname and birthdate who had been enrolled in a particular year. This generated 517 pairs of whom 267 pairs shared the same address, 8 pairs repeated the examination and were listed twice, and the remaining 242 pairs were at different addresses. Each of the 509 
prospective twin pairs was contracted to see if they were twins, and whether they were willing to cooperate in the study. No contact could be established with five pairs. Of the 242 pairs at different addresses only two pairs were found to be twins. Our final sample for which TAE and ASAT results were available thus consisted of 264 pairs of twins (41 $\mathrm{MZ}$ males, $55 \mathrm{MZ}$ females, $52 \mathrm{DZ}$ males, $38 \mathrm{DZ}$ females, $78 \mathrm{DZ}$ opposite- sex). Because many subjects were only attempted by a small number of candidates, we have analysed only those subjects where at least 90 twin pairs were concordant for subject choice, (English, Geography, Mathematics I and Biology), which is probably the lower limit for the useful application of the statistical methods employed below (Martin et al., 1978).

Diagnosis of the zygosity of same-sex pairs was based on their response to the following questions:

1. As children were you and your twin mistaken by people who knew you?

(a) Frequently

(b) Sometimes

(c) Rarely

2. "Non-identical twins are no more alike than ordinary brothers and sisters. Identical twins on the other hand have such a strong resemblance to each other in stature, colouring, features of the face, etc. that people often mistake one for the other" Having read the above statement, do you think you are?

\title{
(a) Identical \\ (b) Non-identical
}

This method of zygosity diagnosis has been found by other workers (Cederlöf et al., 1961; Nichols and Bilbro, 1966; Martin and Martin, 1975; Kasriel and Eaves, 1976) to be about 95 per cent correct, approximately the same reliability as obtained by typing for the most common six or seven blood group polymorphisms.

\begin{abstract}
RESULTS
Sampling

Ideally one would like to analyse the data separately for each year but the numbers are too small to make this practicable. It is necessary, therefore, to pool the results of twins over years, first checking that their means and variances are homogeneous. To assess any overall significant differences over years in the means of the TAE and ASAT results for twins (Table 1), separate one-way analyses of variance were performed. Multiple comparisons were made using Scheffe's test. With the exception of Mathematics I, where there is a significant difference between the 1976 and 1977 values, there is no significant difference between the subject means for the different years. Homogeneity of the twin total variances across years (Table 1) was tested using the Bartlett-Box F test and no significant differences were found.
\end{abstract}

Since the purpose of a genetical analysis of twin data is to make inferences about the causes of variation in the population, it is important that twins are comparable with non-twin subjects. Table 2 presents the means and variances for the total population of candidates attempting the TAE and ASAT in the years 1976 to 1981 . Although in the majority of cases the twin sample had a higher mean than that of the total population, the differences were not great. Differences in variances were similarly small. We are thus justified in generalising the results from the twin sample to the population of TAE and ASAT candidates.

Before fitting models to explain trait variation it is important to test whether the individuals in the $\mathrm{MZ}$ and $\mathrm{DZ}$ groups have been drawn at random from the same population by testing whether the subgroup means and variances are equal. Table 3 
TABLE 1

EXAMINATION RESULTS FOR TWIN INDIVIDUALS By SUBJECT AND YEAR

\begin{tabular}{|c|c|c|c|c|c|c|c|c|c|}
\hline \multirow[b]{2}{*}{ Subject } & & \multicolumn{7}{|c|}{ Year } & \multirow{2}{*}{$\begin{array}{l}\text { All years } \\
\text { combined }\end{array}$} \\
\hline & & 1975 & 1976 & 1977 & 1978 & 1979 & 1980 & 1981 & \\
\hline ASAT Arts & $\begin{array}{l}\text { Mean } \\
\text { SD } \\
N\end{array}$ & N/A & $\begin{array}{c}26 \cdot 27 \\
8 \cdot 61 \\
93\end{array}$ & $\begin{array}{c}25 \cdot 80 \\
7 \cdot 85 \\
76\end{array}$ & $\begin{array}{c}33 \cdot 24 \\
8 \cdot 65 \\
74\end{array}$ & $\begin{array}{c}28 \cdot 01 \\
7 \cdot 35 \\
69\end{array}$ & $\begin{array}{c}30 \cdot 91 \\
7 \cdot 10 \\
97\end{array}$ & $\begin{array}{c}29 \cdot 20 \\
7 \cdot 80 \\
61\end{array}$ & $\begin{array}{c}28 \cdot 40 \\
8 \cdot 55 \\
470\end{array}$ \\
\hline ASAT Science & $\begin{array}{l}\text { Mean } \\
\text { SD } \\
\mathbf{N}\end{array}$ & N/A & $\begin{array}{c}31 \cdot 50 \\
7 \cdot 07 \\
93\end{array}$ & $\begin{array}{c}28.95 \\
7.82 \\
76\end{array}$ & $\begin{array}{c}25 \cdot 84 \\
8 \cdot 18 \\
74\end{array}$ & $\begin{array}{c}29 \cdot 36 \\
9.65 \\
69\end{array}$ & $\begin{array}{c}36 \cdot 55 \\
7 \cdot 63 \\
97\end{array}$ & $\begin{array}{c}31.67 \\
9.02 \\
61\end{array}$ & $\begin{array}{c}30.95 \\
8.81 \\
470\end{array}$ \\
\hline ASAT Total & $\begin{array}{l}\text { Mean } \\
\text { SD } \\
N\end{array}$ & N/A & $\begin{array}{c}57 \cdot 76 \\
14 \cdot 38 \\
93\end{array}$ & $\begin{array}{c}51 \cdot 75 \\
13.82 \\
76\end{array}$ & $\begin{array}{c}59.08 \\
15.96 \\
74\end{array}$ & $\begin{array}{c}57 \cdot 38 \\
15 \cdot 89 \\
69\end{array}$ & $\begin{array}{c}67 \cdot 45 \\
13 \cdot 80 \\
97\end{array}$ & $\begin{array}{c}60 \cdot 87 \\
15 \cdot 01 \\
61\end{array}$ & $\begin{array}{c}59 \cdot 35 \\
11 \cdot 48 \\
470\end{array}$ \\
\hline English & $\begin{array}{l}\text { Mean } \\
\text { SD } \\
\mathbf{N}\end{array}$ & $\begin{array}{c}60.99 \\
16.00 \\
44\end{array}$ & $\begin{array}{c}55 \cdot 77 \\
14 \cdot 93 \\
94\end{array}$ & $\begin{array}{c}57 \cdot 15 \\
14 \cdot 10 \\
73\end{array}$ & $\begin{array}{c}56.13 \\
13.05 \\
69\end{array}$ & $\begin{array}{c}55 \cdot 30 \\
15 \cdot 71 \\
69\end{array}$ & $\begin{array}{c}56 \cdot 26 \\
15 \cdot 33 \\
91\end{array}$ & $\begin{array}{c}60 \cdot 18 \\
13 \cdot 59 \\
59\end{array}$ & $\begin{array}{c}57 \cdot 03 \\
14 \cdot 73 \\
499\end{array}$ \\
\hline Geography & $\begin{array}{l}\text { Mean } \\
\text { SD } \\
\text { N }\end{array}$ & $\begin{array}{c}53 \cdot 62 \\
15 \cdot 17 \\
20\end{array}$ & $\begin{array}{c}53 \cdot 87 \\
15 \cdot 80 \\
58\end{array}$ & $\begin{array}{c}51 \cdot 40 \\
14 \cdot 50 \\
44\end{array}$ & $\begin{array}{c}55 \cdot 50 \\
13 \cdot 00 \\
40\end{array}$ & $\begin{array}{c}51 \cdot 52 \\
15 \cdot 47 \\
35\end{array}$ & $\begin{array}{c}53 \cdot 44 \\
15 \cdot 75 \\
43\end{array}$ & $\begin{array}{c}55 \cdot 00 \\
11 \cdot 12 \\
29\end{array}$ & $\begin{array}{c}53 \cdot 44 \\
14 \cdot 56 \\
269\end{array}$ \\
\hline Mathematics I & $\begin{array}{l}\text { Mean } \\
\text { SD } \\
\text { N }\end{array}$ & $\begin{array}{c}57 \cdot 24 \\
12 \cdot 35 \\
22\end{array}$ & $\begin{array}{c}61 \cdot 81 \\
11 \cdot 39 \\
45\end{array}$ & $\begin{array}{c}51 \cdot 44 \\
12.68 \\
43\end{array}$ & $\begin{array}{c}56 \cdot 35 \\
13 \cdot 25 \\
39\end{array}$ & $\begin{array}{c}59.85 \\
13.93 \\
35\end{array}$ & $\begin{array}{c}57 \cdot 79 \\
15 \cdot 84 \\
63\end{array}$ & $\begin{array}{c}60 \cdot 32 \\
13 \cdot 20 \\
39\end{array}$ & $\begin{array}{c}57 \cdot 82 \\
13 \cdot 78 \\
286\end{array}$ \\
\hline Biology & $\begin{array}{l}\text { Mean } \\
\text { SD } \\
\mathbf{N}\end{array}$ & $\begin{array}{c}51 \cdot 11 \\
12 \cdot 45 \\
14\end{array}$ & $\begin{array}{c}54.58 \\
11.63 \\
47\end{array}$ & $\begin{array}{c}51 \cdot 49 \\
15 \cdot 09 \\
46\end{array}$ & $\begin{array}{c}54 \cdot 73 \\
10 \cdot 91 \\
47\end{array}$ & $\begin{array}{c}54 \cdot 08 \\
16 \cdot 35 \\
40\end{array}$ & $\begin{array}{c}55 \cdot 34 \\
12 \cdot 72 \\
45\end{array}$ & $\begin{array}{c}55 \cdot 66 \\
12 \cdot 28 \\
34\end{array}$ & $\begin{array}{c}54 \cdot 09 \\
13 \cdot 16 \\
273\end{array}$ \\
\hline
\end{tabular}

lists the means and variances of the TAE and ASAT scores for the five twin groups. Two-tailed tests and variance ratio tests were performed between $\mathrm{MZ}$ and $\mathrm{DZ}$ means and total variances, separately for males and females. No significant differences in means or total variances in either sex were found.

It is of interest to know whether there is a difference between $\mathrm{MZ}$ and $\mathrm{DZ}$ twins in their concordance for subject choice. Table 4 shows that $M Z$ twins choose more subjects in common than same-sex $\mathrm{DZ}$ twins which suggests that genetic differences influence the choice of subject taken. In his smaller sample of twin pairs Martin (1975) found no significant differences in concordance for subject choice between $M Z$ and $D Z$ same-sex pairs.

Testing hypotheses about the causes of trait variation

Traditional analyses of twin data have done little more than compare the correlations of $\mathrm{MZ}$ and $\mathrm{DZ}$ twins calculate a "heritability" based on some crude formula which is both inefficient, in that it uses only part of the information available, and often inaccurate in that its calculation is based upon a number of untested assumptions. For these reasons Jinks and Fulker (1970) urged the abandonment of such practices and the rigorous application of the hypothesis testing approach of biometrical genetics. Alternative models of trait variation are fitted to between-and within-pairs mean squares by the method of iterative weighted least squares (Eaves and Eysenck, 1975; Martin, 1975, Eaves et al., 1978). A chi-square test of goodness-of-fit, based on expected mean squares calculated from the least squares parameter estimates, then provides a test of the adequacy of each model.

A large difference in the means of males and females will inflate the within pairs 
TABLE 2

Examination Results by Subject and Year for All Candidates

\begin{tabular}{|c|c|c|c|c|c|c|c|c|c|c|c|c|c|c|c|}
\hline \multirow[b]{2}{*}{ Subject } & \multicolumn{3}{|c|}{1977} & \multicolumn{3}{|c|}{1978} & \multicolumn{3}{|c|}{1979} & \multicolumn{3}{|c|}{1980} & \multicolumn{3}{|c|}{1981} \\
\hline & $\mathbf{N}$ & Mean & SD & $\mathbf{N}$ & Mean & SD & $\mathbf{N}$ & Mean & SD & $\mathrm{N}$ & Mean & SD & $\mathrm{N}$ & Mean & SD \\
\hline $\begin{array}{l}\text { ASAT Arts } \\
\text { ASAT Science } \\
\text { ASAT Total } \\
\text { English } \\
\text { Geography } \\
\text { Mathematics I } \\
\text { Biology }\end{array}$ & $\begin{array}{l}8786 \\
8786 \\
8786 \\
8786 \\
3853 \\
3849 \\
4490\end{array}$ & $\begin{array}{l}23 \cdot 5 \\
29 \cdot 5 \\
53 \cdot 0 \\
54 \cdot 9 \\
52 \cdot 4 \\
54 \cdot 6 \\
52 \cdot 3\end{array}$ & $\begin{array}{r}8.5 \\
7.1 \\
13.9 \\
15 \cdot 2 \\
14.5 \\
14.2 \\
13.8\end{array}$ & $\begin{array}{l}8612 \\
8612 \\
8612 \\
8703 \\
3810 \\
4072 \\
4626\end{array}$ & $\begin{array}{l}26 \cdot 3 \\
34 \cdot 7 \\
61 \cdot 0 \\
57 \cdot 7 \\
54 \cdot 3 \\
55 \cdot 6 \\
53 \cdot 8\end{array}$ & $\begin{array}{r}8.0 \\
7.7 \\
14 \cdot 3 \\
13.8 \\
13.5 \\
14.8 \\
13.3\end{array}$ & $\begin{array}{l}8714 \\
8714 \\
8714 \\
8803 \\
3823 \\
4134 \\
4579\end{array}$ & $\begin{array}{l}27 \cdot 7 \\
27 \cdot 8 \\
55 \cdot 5 \\
56 \cdot 4 \\
53 \cdot 3 \\
55 \cdot 7 \\
52 \cdot 9\end{array}$ & $\begin{array}{r}8 \cdot 8 \\
7 \cdot 0 \\
14 \cdot 1 \\
15 \cdot 0 \\
14 \cdot 3 \\
14 \cdot 5 \\
14 \cdot 0\end{array}$ & $\begin{array}{l}8768 \\
8768 \\
8768 \\
8882 \\
3730 \\
4128 \\
4509\end{array}$ & $\begin{array}{l}35 \cdot 0 \\
31.0 \\
65.9 \\
56 \cdot 7 \\
52.9 \\
57 \cdot 1 \\
53.6\end{array}$ & $\begin{array}{r}8.7 \\
6.8 \\
14.0 \\
15.0 \\
14.6 \\
14.1 \\
14.0\end{array}$ & $\begin{array}{l}8678 \\
8678 \\
8678 \\
8765 \\
3525 \\
4136 \\
3917\end{array}$ & $\begin{array}{l}28 \cdot 8 \\
28 \cdot 1 \\
56 \cdot 9 \\
56 \cdot 3 \\
53 \cdot 1 \\
56 \cdot 8 \\
53 \cdot 1\end{array}$ & $\begin{array}{r}10.1 \\
7.4 \\
15.5 \\
14.9 \\
14.5 \\
13.7 \\
14.0\end{array}$ \\
\hline
\end{tabular}

TABLE 3

Subject Means, Variances and Number of Individuals by Twin Type, Summed OVer Years

\begin{tabular}{|c|c|c|c|c|c|c|c|c|c|c|c|c|c|c|c|}
\hline \multirow[b]{2}{*}{ Subject } & \multicolumn{3}{|c|}{ MZ Males } & \multicolumn{3}{|c|}{ MZ Females } & \multicolumn{3}{|c|}{ DZ Males } & \multicolumn{3}{|c|}{ DZ Females } & \multicolumn{3}{|c|}{ DZ Opposite-Sex } \\
\hline & $\mathbf{N}$ & Mean & Variance & $\mathbf{N}$ & Mean & Variance & $\mathbf{N}$ & Mean & Variance & $\mathbf{N}$ & Mean & Variance & $\mathbf{N}$ & Mean & Variance \\
\hline ASAT Arts & 66 & $28 \cdot 06$ & $72 \cdot 93$ & 100 & 29.06 & 77.44 & 98 & $27 \cdot 84$ & $81 \cdot 54$ & 66 & $26 \cdot 76$ & $59 \cdot 29$ & 140 & $29 \cdot 26$ & 69.89 \\
\hline ASAT Science & 66 & $29 \cdot 88$ & $\begin{array}{r}80.10 \\
189.61\end{array}$ & 100 & 31.77 & $\begin{array}{r}73.27 \\
161.54\end{array}$ & 98 & $\begin{array}{l}30 \cdot 41 \\
53.00\end{array}$ & $\begin{array}{r}93.51 \\
238.30\end{array}$ & 66 & $\begin{array}{r}29.99 \\
59.25\end{array}$ & $\begin{array}{r}67.24 \\
188.79\end{array}$ & 140 & $\begin{array}{l}31.69 \\
57.58\end{array}$ & $\begin{array}{r}73 \cdot 10 \\
240.55\end{array}$ \\
\hline $\begin{array}{l}\text { English } \\
\text { Geography }\end{array}$ & $\begin{array}{l}80 \\
45\end{array}$ & $\begin{array}{l}52 \cdot 11 \\
50 \cdot 92\end{array}$ & $\begin{array}{l}189 \cdot 61 \\
186.05\end{array}$ & $\begin{array}{r}103 \\
53\end{array}$ & $\begin{array}{l}61.37 \\
54.71\end{array}$ & $\begin{array}{l}161 \cdot 54 \\
201 \cdot 64\end{array}$ & $\begin{array}{l}95 \\
52\end{array}$ & $\begin{array}{l}53.90 \\
51.74\end{array}$ & $\begin{array}{l}238 \cdot 39 \\
243 \cdot 67\end{array}$ & $\begin{array}{l}72 \\
42\end{array}$ & $\begin{array}{l}39.23 \\
53.84\end{array}$ & $\begin{array}{l}188 \cdot 79 \\
244 \cdot 30\end{array}$ & $\begin{array}{r}149 \\
77\end{array}$ & $\begin{array}{l}57.58 \\
54.96\end{array}$ & $\begin{array}{l}240 \cdot 25 \\
197 \cdot 40\end{array}$ \\
\hline Mathematics I & 36 & 55.70 & 165.64 & 61 & 58.87 & 254.40 & 50 & $58 \cdot 10$ & $243 \cdot 36$ & 45 & 55.06 & 103.84 & 94 & $59 \cdot 14$ & 169.78 \\
\hline Biology & 24 & $51 \cdot 70$ & 137.59 & 63 & 54.96 & $138 \cdot 30$ & 43 & $51 \cdot 48$ & $199 \cdot 37$ & 54 & $54 \cdot 48$ & 196.00 & 89 & $55 \cdot 15$ & 182.25 \\
\hline
\end{tabular}


TABLE 4

CONCORDANCE OF SUBJeCt CHOICE FOR Different TYPeS OF TWINS

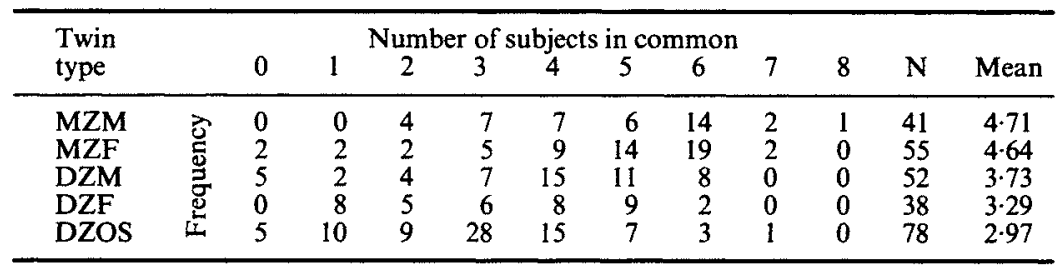

mean squares (WMS) of DZ opposite-sex pairs. In every case except ASAT Science, female twins have a higher mean score than males and so the DZ opposite-sex within pairs mean squares have been corrected for these sex differences (Clark et al., 1980). The mean squares and their degrees of freedom for each variable are shown in Table 5.

A model for variation in $\mathrm{MZ}$ and $\mathrm{DZ}$ mean squares is shown in Table $6 . \mathrm{E}_{1}$ is the environmental variance within families, specific to the individual and shared with no one else, not even members of the same family; it also includes measurement error. $\mathrm{E}_{2}$,

TABLE 5

Observed Mean Squares Between (b) and Within (w) Pairs Used in Model Fitting

\begin{tabular}{|c|c|c|c|c|c|c|c|c|c|c|c|c|}
\hline Statistic & df & $\begin{array}{c}\text { ASAT } \\
\text { Arts }\end{array}$ & $\mathrm{df}$ & $\begin{array}{l}\text { ASAT } \\
\text { Science }\end{array}$ & $\mathrm{df}$ & English & df & Geography & df & Mathematics & df & Biology \\
\hline $\mathrm{MZM}_{\mathrm{b}}$ & 32 & $129 \cdot 93$ & 32 & 139.78 & 39 & $324 \cdot 46$ & 17 & $290 \cdot 46$ & 13 & $302 \cdot 77$ & 5 & $165 \cdot 39$ \\
\hline $\mathrm{MZM}_{\mathrm{w}}$ & 33 & $17 \cdot 70$ & 33 & $22 \cdot 36$ & 40 & $58 \cdot 36$ & 18 & $48 \cdot 81$ & 14 & $60 \cdot 22$ & 6 & $53 \cdot 70$ \\
\hline $\mathrm{MZF}_{\mathrm{b}}$ & 48 & $137 \cdot 00$ & 48 & $124 \cdot 57$ & 49 & $247 \cdot 64$ & 24 & $308 \cdot 01$ & 24 & $289 \cdot 77$ & 27 & $232 \cdot 50$ \\
\hline $\mathrm{MZF}_{\mathrm{w}}$ & 49 & $17 \cdot 86$ & 49 & $18 \cdot 48$ & 50 & $65 \cdot 70$ & 25 & 92.59 & 25 & $204 \cdot 49$ & 28 & $58 \cdot 60$ \\
\hline $\mathrm{DZM}_{\mathrm{b}}$ & 48 & $131 \cdot 51$ & 48 & 154.85 & 45 & 354.49 & 19 & $471 \cdot 75$ & 16 & 316.92 & 13 & 218.53 \\
\hline $\mathrm{DZM}_{w}$ & 49 & $32 \cdot 55$ & 49 & $33 \cdot 49$ & 46 & $127 \cdot 33$ & 20 & $69 \cdot 59$ & 17 & $135 \cdot 26$ & 14 & $62 \cdot 34$ \\
\hline $\mathrm{DZF}_{\mathrm{b}}$ & 31 & $84 \cdot 27$ & 31 & 94.09 & 34 & 228.86 & 14 & $250 \cdot 73$ & 14 & $183 \cdot 76$ & 19 & $329 \cdot 75$ \\
\hline $\mathrm{DZF}_{\mathrm{w}}$ & 32 & $36 \cdot 00$ & 32 & $37 \cdot 42$ & 35 & 155.98 & 15 & 210.97 & 15 & $57 \cdot 34$ & 20 & $75 \cdot 61$ \\
\hline $\mathrm{DZO}_{\mathrm{b}}$ & 67 & $89 \cdot 88$ & 67 & $101 \cdot 29$ & 70 & 274.58 & 21 & $242 \cdot 18$ & 26 & $270 \cdot 73$ & 25 & $221 \cdot 34$ \\
\hline $\mathrm{DZO}_{\mathrm{w}}^{\mathrm{a}}$ & 67 & $48 \cdot 06$ & 67 & $46 \cdot 61$ & 70 & $177 \cdot 21$ & 21 & $156 \cdot 42$ & 26 & 95.59 & 25 & 127.09 \\
\hline
\end{tabular}

${ }^{\text {a }}$ Corrected for mean differences between males and females

TABLE 6

Simple Model for Twin MEAn SQuares

\begin{tabular}{lllll}
\hline \multicolumn{1}{c}{ Mean squares } & & $\mathrm{E}_{1}$ & $\mathrm{E}_{2}$ & $\mathrm{~V}_{\mathrm{A}}$ \\
\hline \multirow{2}{*}{ MZ female } & Between & 1 & 2 & 2 \\
& Within & 1 & 0 & 0 \\
MZ male & Between & 1 & 2 & 2 \\
& Within & 1 & 0 & 0 \\
DZ female & Between & 1 & 2 & $\frac{3}{2}$ \\
& Within & 1 & 0 & $\frac{1}{2}$ \\
DZ male & Between & 1 & 2 & $\frac{3}{2}$ \\
& Within & 1 & 0 & $\frac{1}{2}$ \\
DZ opposite-sex & Between & 1 & 2 & $\frac{3}{2}$ \\
& Within & 1 & 0 & $\frac{1}{2}$ \\
\hline
\end{tabular}


the between families environmental component, estimates sources of environmental variance shared by both members of a twin pair but differing between pairs, including such factors as cultural differences and parental rearing practices. Since the great majority of our twin pairs attended the same school $\mathrm{E}_{2}$ would also include the effect of any differences between school environments.

$\mathrm{V}_{\mathrm{A}}$ is that part of genetic variation due to the additive effect of genes in the absence of assortative mating. If assortative mating (the tendency of like to marry like, as measured by the correlation between husbands and wives) occurs for a given character it will increase the additive genetic variance between pairs but in proportions such that it is completely confounded with estimates of $\mathrm{E}_{2}$ in twin data. Any variation attributed to this source must therefore be interpreted with caution since it could arise from assortative mating, shared environment or both.

In the interests of parsimony the simplest model should be fitted first and more complicated models considered only if simpler ones fail or a significant improvement in fit is obtained by addition of extra parameters. Accordingly we first fit a simple environmental model containing $\mathrm{E}_{1}$ alone. Failure of this model indicates that there is significant variation between families. A model incorporating $E_{1}$ and $E_{2}$ tests whether the between-families variation is entirely environmental in origin, whilst an alternative incorporating $E_{1}$ and $V_{A}$ tests whether it is entirely genetic. If both two parameter models fail, a model including $\mathrm{E}_{1}, \mathrm{E}_{2}$ and $\mathrm{V}_{\mathrm{A}}$ must be considered.

Results of fitting models to explain trait variation

The results of fitting models of variation to the TAE and ASAT results are summarised in Table 7. With the exception of Mathematics $I$, a model $\left(E_{1}\right)$ postulating

TABLE 7

Summary of Results of Model-Fitting. Heritability Estimates Given Where Appropriate

\begin{tabular}{|c|c|c|c|c|c|c|c|}
\hline Subject & $\begin{array}{l}\text { Data } \\
\text { Set } †\end{array}$ & $\hat{\mathbf{E}}_{1}$ & $\hat{\mathbf{E}}_{2}$ & $\nabla_{A}$ & df & $\chi^{2}$ & $\hat{h}^{2}$ \\
\hline ASAT Arts & 10 & $\begin{array}{l}32 \cdot 29^{* * *} \\
17 \cdot 03^{* * *} \\
17 \cdot 67^{* * *}\end{array}$ & $\frac{40 \cdot 67^{* * *}}{9 \cdot 04}$ & $\begin{array}{l}-\overline{4 \cdot 68^{* * *}} \\
45 \cdot 99^{* * *}\end{array}$ & $\begin{array}{l}8 \\
8 \\
7\end{array}$ & $\begin{array}{l}20.91 * * \\
6.01 \\
5.26\end{array}$ & $0.76 \pm 0.04$ \\
\hline ASAT Science & 10 & $\begin{array}{l}33 \cdot 06^{* * *} \\
18 \cdot 73^{* * *} \\
20 \cdot 14^{* * *}\end{array}$ & $\frac{44 \cdot 50^{* * *}}{17 \cdot 81^{*}}$ & 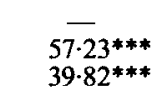 & $\begin{array}{l}8 \\
8 \\
7\end{array}$ & $\begin{array}{c}16 \cdot 26^{*} \\
9 \cdot 31 \\
5 \cdot 99\end{array}$ & $\begin{array}{l}0.75 \pm 0.04 \\
0.51 \pm 0.14\end{array}$ \\
\hline English & 10 & $\begin{array}{r}121 \cdot 70^{* * *} \\
68 \cdot 29^{* * *} \\
64 \cdot 73^{* * *}\end{array}$ & $\begin{array}{c}82 \cdot 04^{* * * *} \\
-27 \cdot 41\end{array}$ & $\begin{array}{l}-\overline{140 \cdot 70^{* * *}} \\
170 \cdot 10^{* * *}\end{array}$ & $\begin{array}{l}8 \\
8 \\
7\end{array}$ & $\begin{array}{l}22 \cdot 23^{* *} \\
7 \cdot 97 \\
7 \cdot 04\end{array}$ & $0.67 \pm 0.05$ \\
\hline Geography & 10 & $\begin{array}{r}111 \cdot 50^{* * *} \\
74.06^{* * *} \\
76 \cdot 66^{* * *}\end{array}$ & $\frac{101 \cdot 60^{* * *}}{23 \cdot 42}$ & $\begin{array}{l}\overline{140 \cdot 30^{* * *}} \\
115 \cdot 40^{*}\end{array}$ & $\begin{array}{l}8 \\
8 \\
7\end{array}$ & $\begin{array}{l}15 \cdot 57^{*} \\
11 \cdot 12 \\
11.08\end{array}$ & $0.65 \pm 0.08$ \\
\hline Mathematics I & $\mathbf{M}$ & $\begin{array}{c}198 \cdot 70^{* * *} \\
149 \cdot 30^{* * *} \\
158 \cdot 80^{* * *} \\
179 \cdot 80^{* * *} \\
202 \cdot 50^{* * *} \\
101 \cdot 40^{* * *} \\
60 \cdot 86^{* *} \\
61 \cdot 72^{* *}\end{array}$ & $\begin{array}{c}5 \overline{5} \overline{70} \\
\overline{266 \cdot 70^{* *}} \\
\overline{1} \\
104 \cdot 60^{* *} \\
\overline{11 \cdot 19}\end{array}$ & $\begin{array}{c}- \\
\overline{-} \\
39 \cdot 70 \\
-235 \cdot 10 \\
- \\
\overline{-} \\
147 \cdot 40^{* * *} \\
136 \cdot 20\end{array}$ & $\begin{array}{l}3 \\
2 \\
2 \\
1 \\
3 \\
2 \\
2 \\
1\end{array}$ & $\begin{array}{l}6 \cdot 37 \\
5 \cdot 34 \\
5 \cdot 23 \\
2 \cdot 41 \\
8 \cdot 54^{*} \\
2 \cdot 11 \\
0.28 \\
0.24\end{array}$ & $\begin{array}{l}0.71 \pm 0.12 \\
0.65 \pm 0.45\end{array}$ \\
\hline Biology & 10 & $\begin{array}{l}80 \cdot 92^{* * *} \\
54 \cdot 32^{* * *} \\
59 \cdot 90^{* * *}\end{array}$ & $\begin{array}{l}81 \cdot 70^{* * *} \\
4 \overline{1 \cdot 17}\end{array}$ & $\begin{array}{l}107 \cdot 40^{* * *} \\
62 \cdot 94\end{array}$ & $\begin{array}{l}8 \\
8 \\
7\end{array}$ & $\begin{array}{l}7 \cdot 52 \\
5 \cdot 94 \\
5 \cdot 12\end{array}$ & $\begin{array}{l}0.66 \pm 0.08 \\
0.38 \pm 0.26\end{array}$ \\
\hline
\end{tabular}

$\uparrow$ Results are shown for females (F), males (M) and females, males and opposite-sex (10) where appropriate. $\begin{array}{ll}\text { Tesults are shown for females }(\mathrm{F}) \text {, males }(\mathrm{M}) \text { and } \\ \mathrm{P}<0.05 & * * \mathrm{P}<0.01\end{array}$ 
that all variation was due to individual environmental experiences failed badly and is omitted from the table. For all subjects (except Mathematics I) the same model was appropriate for both sexes, so models were fitted to all ten mean squares. For Mathematics I, models have been fitted separately to the four mean squares for females and the four mean squares for males.

For ASAT Arts, English and Geography, a purely environmental $\left(E_{1} E_{2}\right)$ model fails to account for the data while the simple genetic model $\left(E_{1} V_{A}\right)$ gives a good fit. For these three variables no further reductions in chi-square are obtained with the addition of an $E_{2}$ parameter to the model.

Although the $\mathrm{E}_{1} \mathrm{~V}_{\mathrm{A}}$ model provides an adequate description of the ASAT Science data, addition of the $E_{2}$ parameter results in significant estimates of all three sources of variation indicating that the $\mathrm{E}_{1} \mathrm{E}_{2} \mathrm{~V}_{\mathrm{A}}$ model is most appropriate. This is despite the fact that the improvement in chi-square over the $E_{1} V_{A}$ model is only of marginal significance $\left(\chi_{1}^{2}=3.32,0.05<\mathrm{P}<0.10\right)$.

For Mathematics $\mathrm{I}$, the $\mathrm{E}_{1}$ model is able to describe adequately the data in females, indicating that there is no significant between families variation to be accounted for and that all variance is due to individual environment experiences. Although in males the data are consistent with both an $E_{1} E_{2}$ or $E_{1} V_{A}$ model, the genetical model gives a better fit. When all three parameters are fitted, non-significant estimates are obtained for both $\hat{\mathrm{E}}_{2}$ and $\hat{\mathrm{V}}_{\mathrm{A}}$, and $\hat{\mathrm{E}}_{2}$ accounts for a trivial proportion of the variance (about 5 per cent).

For Biology, as with Mathematics 1 in males, there is little to choose between the $E_{1} E_{2}$ and $E_{1} V_{A}$ models, although the latter gives a slightly better fit. Again, where all three parameters are fitted, non-significant estimates are obtained for $\hat{E}_{2}$ and $\hat{V}_{A}$, although $\hat{\mathrm{E}}_{2}$ accounts for about 25 per cent of the variation.

\section{The causes of covariation between ASAT Arts and Science scores}

We have detected genetic variation for performance in all subjects (with the possible exception of Mathematics in females) and we may ask whether each subject is probing the same variation in a different way or whether some gene action is specific to certain subjects. Since not all twins have sat examinations in the same subjects we cannot employ the most elegant methods to answer this question (Martin and Eaves, 1977; Martin et al., 1984). However, all twins since 1976 have taken the ASAT Arts and Science tests. The correlation between scores for these two tests is 0.58 and we wish to know the basis of this covariation. The procedure is described in detail in Eaves et al., $(1978$, pp. 301-304). The first step is to calculate the $4 \times 4$ covariance matrices between the Arts and Science scores for Twin 1 and Twin 2, one matrix for each of the five twin types. Models are then fitted to the covariance matrices in which the variation and covariation between Arts and Science scores is attributed to various combinations of the three major sources, $E_{1}, E_{2}$ and $V_{A}$. For an $E_{1} E_{2}$ model of variation and covariation a fit of $\chi_{44}^{2}=42.7$ was obtained while the corresponding $E_{1} V_{A}$ model yielded $\chi_{44}^{2}=23.1$. When covariation and variation from all three sources was specified in the model, a fit of $\chi_{41}^{2}=18.2$ resulted. Although none of the models is formally rejected by the chisquare criterion we note that the three source model represents a large and significant improvement over the $\mathrm{E}_{1} \mathrm{E}_{2}$ model $\left(\chi_{3}^{2}=24.5, \mathrm{P}<0.001\right)$ but not over the $\mathrm{E}_{1} \mathrm{~V}_{\mathrm{A}}$ model $\left(\chi_{3}^{2}=4.9,0.10<P<0.20\right)$. We conclude that genetic factors are an important cause of covariation between Arts and Science performance but that $E_{2}$ is not. Estimates for the variance components for Arts and Science are similar to those from the univariate model fitting in Table 7 and the components of covariance for the three source model are $\mathrm{V}_{\mathrm{A}}=31.0 \pm 8.5, \mathrm{E}_{1}=6.9 \pm 2.2, \mathrm{E}_{2}=5.7 \pm 7.9$. These translate into genetic, $\mathrm{E}_{1}$ and $\mathrm{E}_{2}$ correlations of $0.73,0.37$ and 0.43 respectively. 


\section{DISCUSSION}

Twins taking the Tertiary Admission Examination in Western Australia from 1975 to 1981 appear typical of the other candidates in their cohorts. There is no indication of substantial heterogeneity in the twin samples between years nor between zygosity groups after pooling over years. Greater concordance between MZ than between $\mathrm{DZ}$ twins in the choice of examination subjects suggests that there is a genetic component in this choice.

Our analysis of the causes of variation in examination performance demonstrates that a significant proportion of this variation in each subject (except perhaps Mathematics in females and Biology) must be due to genetic factors. A model in which we sought to explain all variation in terms of environmental influences unique to the individual $\left(E_{1}\right)$ or shared with co-twins $\left(E_{2}\right)$ was firmly rejected. A model invoking additive genetic variation, however, gave a good fit in each case and no further significant improvement in fit could be obtained by allowing for shared environmental variation as well. Our results are very similar to those of other studies of examination performance on younger twin samples in South Australia (Martin, 1975) and Brazil (Salzano and Rao, 1976).

None of the above is to suggest that the only source of variation between pairs is due to the segregation of additive genes, but simply that our study, although bigger than most, does not have the statistical power to detect any other source. It has been shown that very much larger samples of twins than those available here are needed to reliably detect $V_{A}$ and $E_{2}$ when both sources are making substantial contributions to trait variation (Martin et al., 1978). The inability to resolve the causes of variation in Mathematics and Biology is largely due to the very small numbers of twins who were candidates for examination in those subjects.

A further complication in the interpretation of our results is that our twin sample is not typical of the population cohort as a whole but only of that portion of the cohort who were candidates for the TAE. This portion is only 34 per cent of the age cohort and we assume, in the absence of evidence to the contrary, that they represent the top 34 per cent from an underlying normal distribution of ability. Martin and Wilson (1982) have considered the bias that such truncate selection of twin samples will introduce to the calculation of genetic and environmental components of variation. Correlation coefficients estimated from such truncate samples will be biased downwards from their true population values, but if the trait has a genetic component then this bias will not be in the same proportion in $\mathrm{MZ}$ and DZ twins. In the present case let us take rough median values from our data of $r_{m z}=0.70$ and $r_{d z}=0.35$ which are consistent with a heritability of 0.70 and no $\mathrm{E}_{2}$ variance. If we now interpolate in Figure 1 of Martin and Wilson (1982) using 66 per cent (100-34) sample truncation, we would obtain approximate estimates for the underlying ability trait in the population of $R_{m z}=0.87$ and $R_{d z}=0.65$. These putative population correlations would now be consistent with a random mating heritability of 0.44 and an $E_{2}$ component of 0.43 .

Before assuming that this correction for truncate sampling has reinstated shared family environment to an important place in the aetiology of individual differences in educational achievement, we must remember that what we have estimated above as " $E_{2}$ " also contains any additional genetic variance arising from assortative mating. There is certainly detectable covariation between spouses for educational achievement, estimates of the marital correlation ranging from about 0.3 to 0.7 (Jencks, 1972; Heath et al., 1984). Even under moderate levels of assortative mating the extra additive genetic variation generated by assortative mating is considerable (Eaves et al., 1978). Taking a median correlation between husbands and wives for educational attainment of 0.4 , it can be calculated (see Martin, 1978) that more than half the variance attributed to " $\mathrm{E}_{2}$ " is genetic variance arising from assortative mating. 
Taking all the above considerations into account, we would conclude that the total proportion of variance due to genetic factors is in the range of 60-70 per cent and that shared environmental influences (home background, shared schooling etc.) account for no more than 20 per cent of the total variance in examination performance. These proportions are very similar to the best estimates for the causes of variation in IQ (Fulker and Eysenck, 1979) and suggest that a distinction between IQ tests and tests of scholastic achievement on the basis of their heritabilities (e.g. Husén, 1960; Jensen, 1969 ) is not justified (cf. Willerman et al., 1977).

We have been able to show that much of the covariation between ASAT Arts and Science scores is genetic in origin and that environmental factors contribute little to their correlation. The fact that the genetic correlation is only 0.73 indicates that, in addition to the common genetic factor underlying covariation, there are some differences in the genetic effects on the two traits. There is thus some support for the view that it is principally genetic factors which determine general ability and mainly (but not exclusively) environmental factors which channel it into proficiency in a particular subject area such as arts or science.

The finding of both common and specific genetic variation for performance in different tests is in line with the results from multivariate analyses of other twin studies of multiple abilities (Martin, 1975; Martin and Eaves, 1977; Martin et al., 1984). A feature of the latter two studies, however, was that the " $E_{2}$ " correlations between variables were all near unity. Eaves et al., (1984) have argued that this is a consequence of assortative mating and/or cultural transmission operating on a latent variable which is a linear combination of the measured ability variables. In the present study we suggest that the low value of the " $E_{2}$ " correlation is due to the combined effects of truncate sampling and the low power available to distinguish between additive genetic variation and other sources of variation between families.

The individual environmental correlation of 0.37 is small but the fact that it is greater than zero suggests that not all $\mathrm{E}_{1}$ variance can be written off as "error". Heritabilities corrected for unreliable "error" variance will, of course, be higher than those quoted above but it is not clear what is gained by this practice, especially since it has been shown that "unreliability" itself may have a genetic component (Eaves and Eysenck, 1976). To the extent that an individual's career prospects are determined by his performance in a single examination, one is interested only in the causes of variation in that single measurement. However, to the extent that examination performance is an imperfect measure of an underlying trait on which, for instance, mate selection is based, heritability of the latent variable may be of more interest.

ACKNOWLEDGMENTS. - We would like to thank the Board of Management of the Tertiary Institutions Services Centre who gave us access to the ASAT and TAE results and Dr. N. A. Goodchild of the Biometrics Unit, University of Western Australia for his supervision of C. E. Gill. We are grateful to Dr. L. J. Eaves and Dr. A. C. Heath for helpful comments on the manuscript. Most of all we are indebted to the twins, without whose co-operation this work would not have been possible.

Correspondence should be addressed to Dr. N. G. Martin, Department of Human Genetics, Medical College of Virginia, Virginia Commonwealth University, PO Box 33; MCV Station, Richmond, Virginia 23298, USA.

\section{REFERENCES}

Cederlóf, R., Friberg, L., Jonsson, E., and KaIJ, L. (1961). Studies on similarity diagnosis in twins with the aid of mailed questionnaires. Acta Genetica et Statistica Medica, 11, 338-362.

Clark, P. Jardine, R., Martin, N. G., Stark, A. E., and Walsh, R. J. (1980). Sex differences in the inheritance of some anthropometric characters in twins. Acta Geneticae Medicae et Gemellologiae, 29, $171-192$. 
EAVES, L. J. (1975). Testing models for variation in intelligence. Heredity, 34, 132-136.

EAves, L. J., and EysenCK, H. J. (1975). The nature of extraversion: a genetical analysis. J. Person. soc. Psychol., 32, 102-112.

EAvEs, L. J., and EYSENCK, H. J. (1976). Genetic and environmental components of inconsistency and unrepeatability in twins' responses to a Neuroticism questionnaire. Behav. Genetics, 6, 145-160.

Eaves, L. J., Heath, A. C., and Martin, N. G. (1984). A note on the generalised effects of assortative mating. Behav. Genetics, 14, 371-376.

Eaves, L. J., LAst, K. A., Young, P. A., and Martin, N. G. (1978). Model-fitting approaches to the analysis of human behaviour. Heredity, 41, 249-320.

FulKER, D. W., and EYSENCK, H. J. (1979). Nature and nurture: heredity. In EYSENCK, H. J. (Ed.), The Structure and Measurement of Intelligence. New York: Springer Verlag.

Heath, A. C., Berg, K., Eaves, L. J., SolaAs, M. H., Sundet, J., Nance, W. E., Corey, L. A., and Magnus, P. (1985). No decline in assortative mating for educational level. Behav. Genetics, 15 , 349-370.

HorN, J. M., Loehlin, J. C., and Willerman, L. (1982). Aspects of the inheritance of intellectual abilities. Behav. Genetics, 12, 479-516.

HusÉN, T. (1960). Abilities of twins. Scand. J. Psychol., 1, 125-135.

JENCKS, C. et al. (1972). Inequality: A Reassessment of the Effect of Family and Schooling in America. Harmondsworth: Penguin.

JENSEN, A. R. (1969). How much can we boost IQ and scholastic achievement? Harvard educ. Rev., 39 , $1-123$.

JINKS, J. L., and Fulker, D. W. (1970). Comparison of the biometrical genetical, MAVA and classical approaches to the analysis of human behaviour. Psychol. Bull., 73, 311-349.

KASRIEL, J., and EAVES, L. (1976). The zygosity of twins: further evidence on the agreement between diagnosis by blood groups and written questionnaires. $J$. biosoc. $S c i ., 8,263-266$.

Loehlin, J. C., and Nichols, R. C. (1976). Heredity, Environment and Personality. Austin: University of Texas Press.

Martin, N. G. (1975). The inheritance of scholastic abilities in a sample of twins. II. Genetical analysis of examination results. Annals of Human Genetics, 39, 219-228.

Martin, N. G. (1978). Genetics of sexual and social attitudes in twins. In Twin Research: Psychology and Methodology. New York: Alan R. Liss.

Methodology. New York: Alan R. Liss.
MARTIN, N. G., and EAves, L. J. (1977). The genetical analysis of covariance structure. Heredity, 38, 79-95. MARTIN, N. G., EAVES, L. J., KEARSEY, M. J., and DAVIES, P. (1978). The power of the classical twin study.
Heredity, 40, 97-116.

MARTIN, N. G., JARDine, R., and EAvEs, L. J. (1984). Is there only one set of genes for different abilities? A reanalysis of the NMSQT data. Behav. Genetics, 14, 355-370.

Martin, N. G., and MARTIN, P. G. (1975). The inheritance of scholastic abilities in a sample of twins. I. Ascertainment of the sample and diagnosis of zygosity. Annals of Human Genetics, 39, 213-218.

MARTIN, N. G., and WILSON, S. R. (1982). Bias in the estimation of heritability from truncated samples of twins. Behav. Genetics, 12, 467-472.

Nichols, R. C., and Bilbro, W. C. (1966). The diagnosis of twin zygosity. Acta Genetica et Statistica Medica, 16, 265-275.

Rao, D. C., Morton, N. E., Elston, R. C., and YeE, S. (1977). Causal analysis of academic performance. Behav. Genetics, 7, 147-159.

Rice, J., Cloninger, C. R., and Reich, T. (1980). Analysis of behavioral traits in the presence of cultural transmission and assortative mating. Behav. Genetics, 10, 73-92.

Salzano, F. M., and RaO, D. C. (1976). Path analysis of aptitude, personality and achievement scores in Brazilian twins. Behav. Genetics, 4, 461-466.

Willerman, L., Horn, J. M., and Loenlin, J. C. (1977). The aptitude-achievement test distinction: A study of unrelated children reared together. Behav. Genetics, 7, 465-470.

(Manuscript received 21st March, 1984) 